OPEN ACCESS

Edited by:

Maurizio Trovato,

Sapienza University of Rome, Italy

Reviewed by:

Donald Becker,

University of Nebraska-Lincoln,

United States

Qiaoquan Liu,

Yangzhou University, China

*Correspondence:

Paulo Arruda

parruda@unicamp.br

Specialty section:

This article was submitted to

Plant Metabolism

and Chemodiversity,

a section of the journal

Frontiers in Plant Science

Received: 17 February 2020

Accepted: 20 April 2020

Published: 21 May 2020

Citation:

Arruda P and Barreto P (2020)

Lysine Catabolism Through

the Saccharopine Pathway: Enzymes and Intermediates Involved in Plant

Responses to Abiotic and Biotic

Stress. Front. Plant Sci. 11:587.

doi: $10.3389 /$ fpls.2020.00587

\section{Lysine Catabolism Through the Saccharopine Pathway: Enzymes and Intermediates Involved in Plant Responses to Abiotic and Biotic Stress}

\author{
Paulo Arruda ${ }^{1,2,3 *}$ and Pedro Barreto $1,2,3$ \\ ${ }^{1}$ Centro de Biologia Molecular e Engenharia Genética, Universidade Estadual de Campinas (UNICAMP), Campinas, Brazil, \\ ${ }^{2}$ Departamento de Genética, Evolução e Bioagentes, Instituto de Biologia, Universidade Estadual de Campinas (UNICAMP), \\ Campinas, Brazil, ${ }^{3}$ Genomics for Climate Change Research Center (GCCRC), Universidade Estadual de Campinas \\ (UNICAMP), Campinas, Brazil
}

The saccharopine pathway (SACPATH) involves the conversion of lysine into $\alpha$-aminoadipate by three enzymatic reactions catalyzed by the bifunctional enzyme lysine-ketoglutarate reductase/saccharopine dehydrogenase $(\mathrm{LKR} / \mathrm{SDH})$ and the enzyme $\alpha$-aminoadipate semialdehyde dehydrogenase (AASADH). The LKR domain condenses lysine and $\alpha$-ketoglutarate into saccharopine, and the SDH domain hydrolyzes saccharopine to form glutamate and $\alpha$-aminoadipate semialdehyde, the latter of which is oxidized to $\alpha$-aminoadipate by AASADH. Glutamate can give rise to proline by the action of the enzymes $\Delta^{1}$-pyrroline-5-carboxylate synthetase (P5CS) and $\Delta^{1}$-pyrroline-5-carboxylate reductase (P5CR), while $\Delta^{1}$-piperideine-6-carboxylate the cyclic form of $\alpha$-aminoadipate semialdehyde can be used by P5CR to produce pipecolate. The production of proline and pipecolate by the SACPATH can help plants face the damage caused by osmotic, drought, and salt stress. AASADH is a versatile enzyme that converts an array of aldehydes into carboxylates, and thus, its induction within the SACPATH would help alleviate the toxic effects of these compounds produced under stressful conditions. Pipecolate is the priming agent of $\mathrm{N}$-hydroxypipecolate (NHP), the effector of systemic acquired resistance (SAR). In this review, lysine catabolism through the SACPATH is discussed in the context of abiotic stress and its potential role in the induction of the biotic stress response.

Keywords: Saccharopine pathway, lysine catabolism, biotic stress, abiotic stress, drought stress

\section{INTRODUCTION}

Plant adaptation to stressful environmental conditions such as drought, salinity, high temperature, and flooding, requires extensive remodeling at the cell, organ, and whole-plant level to sustain structural and metabolic homeostasis. For agriculture, abiotic stress is among the most pressing challenges and, depending on its intensity, frequency, and duration, can cause heavy losses in crop production (Lobell et al., 2011; Ray et al., 2015). 
Plants have evolved multiple mechanisms to adapt to biotic and abiotic stress that involve remodeling organelle structure and metabolic pathways associated with protein, nucleic acids, carbohydrates, lipids, amino acids, phytohormones, cations, and small molecule that modulate stress responses (Van Wallendael et al., 2019). Thus, it is expected that not a single component of this integrated network would be capable of modulating cellular and metabolic responses to alleviate the impact of stressful conditions. On the contrary, the performance of plants subjected to biotic and abiotic stress requires a concerted orchestration of the multiple cellular and metabolic remodeling processes. This review focuses on one aspect of amino acid metabolism, lysine catabolism through the saccharopine pathway (SACPATH), which regulates the accumulation of lysine in seeds (Long et al., 2013; and for reviews, see Yu and Tian, 2018; Zhou et al., 2020 ) and is also involved in abiotic and biotic stress responses (Arruda and Neshich, 2012).

To date, three routes for lysine catabolism have been described in plants: the cadaverine pathway, the SACPATH and the NHP pathway. In the cadaverine pathway, the enzyme lysine decarboxylase converts lysine into the alkaloid cadaverine (Bunsupa et al., 2012). In the SACPATH, that is common to plants, animals, and bacteria, lysine is converted into $\alpha$-aminoadipate by three enzymatic reactions catalyzed by lysine-ketoglutarate reductase/saccharopine dehydrogenase (LKR/SDH) and $\alpha$-aminoadipate semialdehyde dehydrogenase (AASADH) (Markovitzs and Chuang, 1987; Papes et al., 1999; Arruda et al., 2000; Azevedo et al., 2003; Struys and Jakobs, 2010; Neshich et al., 2013). In the NHP pathway, lysine is converted into NHP by three reaction steps catalyzed by the enzymes aminotransferase AGD2-like defense response protein (ALD1), which deaminates lysine into dehydropipecolate (DHP), the reductase systemic acquired resistance-deficient 4 (SARD4), which reduces DHP to pipecolate, and flavin-dependent monooxygenase (FMO1), which N-hydroxylates pipecolate to generate NHP (for a review, see Hartmann and Zeier, 2018). NHP is central to plant immunity due to its role in the activation of systemic acquired resistance (SAR) upon pathogen attack (Hartmann and Zeier, 2018). However, the SACPATH has also recently been shown to give rise to pipecolate in a reaction catalyzed by the enzyme $\Delta^{1}$-pyrroline-5-carboxylate reductase (P5CR), which is critical for proline biosynthesis (Szabados and Savoure, 2009), on $\Delta^{1}$-piperideine-6-carboxylate, the cyclic form of $\alpha$-aminoadipate semialdehyde (Struys and Jakobs, 2010, Struys et al., 2014). This review is not intended to provide a comprehensive overview of lysine catabolism in plants. Instead, it focuses on the SACPATH peculiarities that gives support to its role in the abiotic stress response and its potential contribution to the biotic stress response. Many excellent reviews concerning biotic stress response are available in the literature and may help complement the ideas and concepts presented in this review.

\section{ENZYMES OF THE SACPATH}

The central enzymes of the SACPATH catalyze a transaminationlike reaction involving the enzymes LKR/SDH and AASADH
(Figure 1B). In this pathway, lysine is condensed with $\alpha$-ketoglutarate to generate saccharopine using NADPH as a cofactor. Saccharopine is than hydrolyzed to form $\alpha$-aminoadipate semialdehyde and glutamate using $\operatorname{NAD}(\mathrm{P})^{+}$ as a cofactor. Then, AASADH oxidezes $\alpha$-aminoadioate semialdehyde to $\alpha$-aminoadioate using $\mathrm{NAD}(\mathrm{P})^{+}$as a cofactor. The glutamate generated in the pathway can be metabolized by $\Delta^{1}$-pyrroline-5-carboxylate synthetase (P5CS) and P5CR to produce proline while the cyclic form of $\alpha$-aminoadipate semialdehyde, $\Delta^{1}$-piperideine-6-carboxylate, can be used by P5CR to produce pipecolate (Figure 2). The catalytic properties and subcellular localization of each of these enzymes may help in the understanding of their metabolic role in stress response.

\section{$L K R / S D H$}

The enzyme LKR/SDH was first demonstrated to occur in plants in developing maize endosperm (Arruda et al., 1982; Brochetto-Braga et al., 1992). LKR and SDH belong to a single $\sim 120 \mathrm{kDa}$ bifunctional polypeptide (Gonçalves-Butruille et al., 1996; Gaziola et al., 1997) that localizes in the cytosol (Kemper et al., 1999) in contrasts with the animal LKR/SDH, that localizes in the mitochondria matrix (Markovitzs and Chuang, 1987). Immature endosperms of high-lysine maize mutants, in addition to the bifunctional LKR/SDH polypeptide, also presents a small proportion of an active monofunctional SDH (Pompeu et al., 2006). Monofunctional SDH have also been found in animals (Papes et al., 1999) and in Arabidopsis (Stepansky et al., 2005). A monofunctional SDH have also been detected in Arabidopsis mitochondria (Fuchs et al., 2019). In animals, SDH activity seems to be essential inside mitochondria, as the organelle, in addition to the bifunctional LKR/SDH polypeptide, have significant amounts of a monofunctional SDH (Zhou et al., 2018).

In immature maize and rice endosperm, the enzymatic activity of the LKR domain is activated by $\mathrm{Ca}^{2+}, \mathrm{Mg}^{2+}$, high salt, and osmolytes (Kemper et al., 1998; Gaziola et al., 2000). The LKR domain, when separated from the SDH domain by limited proteolysis, retains its $\mathrm{Ca}^{2+}$ activation. However, it is inhibited by a protein fraction containing SDH polypeptides, suggesting that the SDH domain appears to negatively regulate the activity of the LKR domain (Kemper et al., 1998). The LKR domain but not the SDH domain is also activated by phosphorylation in a lysine-dependent manner (Arruda et al., 2000; Azevedo and Lea, 2001). In plants, the LKR and SDH domains of the bifunctional polypeptide are separated from each other by an $\sim 130$ amino acid interdomain (Kemper et al., 1999; Anderson et al., 2010). Genome sequencing of genes encoding LKR/SDH revealed that, in most plants, the enzyme is encoded by a single gene, with a lone exception reported for poplar, which has two functional genes. Interestingly, transcripts encoding monofunctional LKR and monofunctional SDH have been identified in several plant species (Anderson et al., 2010).

\section{AASADH}

The AASADH enzyme belongs to the superfamily of $\mathrm{NAD}(\mathrm{P})^{+}$dependent aldehyde dehydrogenases (ALDs) that catalyze the conversion of aliphatic and aromatic aldehydes into their corresponding carboxylates (Singh et al., 2012). AASADH can 
A

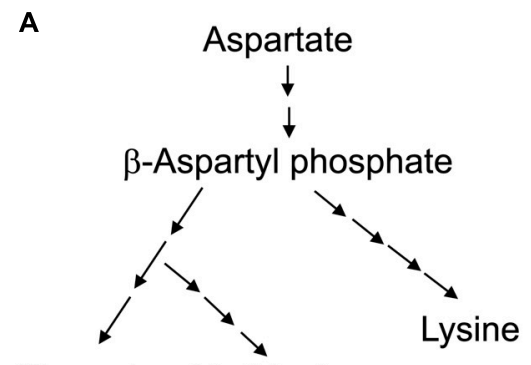

Threonine Methionine

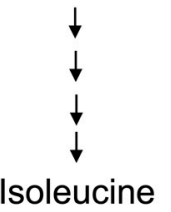

B

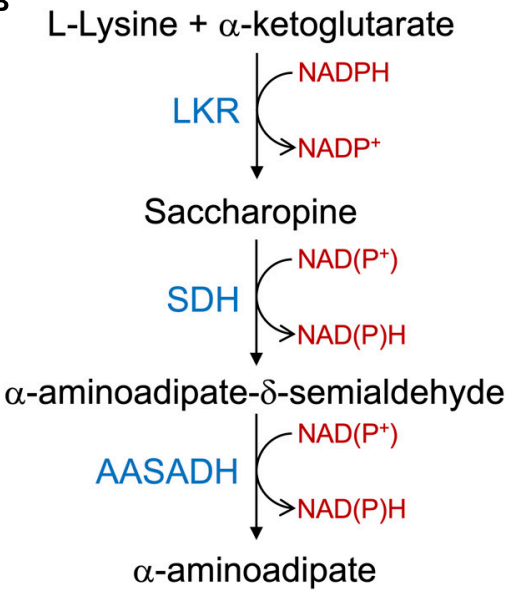

FIGURE 1 | The aspartate pathway for lysine biosynthesis and saccharopine pathway for lysine catabolism. (A) Schematic representation of the aspartate pathway. Only the key biosynthetic steps are represented. Aspartate is converted into $\beta$-aspartyl phosphate that is used in a serial enzymatic step by steps to produce lysine, threonine, methionine and Isoleucine. The aspartate pathway is localized in the plastids and thus lysine needs to be transported to the cytosol and other organelles. (B) The core reactions of the SACPATH involve the conversion of L-lysine into $\alpha$-aminoadipate. The first two steps of the pathway are catalyzed by the bifunctional enzyme LKR/SDH; the LKR domain condenses L-lysine and $\alpha$-ketoglutarate into saccharopine using NADPH as a cofactor and the SDH domain hydrolyzes saccharopine into $\alpha$-aminoadipate semialdehyde and glutamate using $\mathrm{NAD}(\mathrm{P})^{+}$as cofactors. The $\alpha$-aminoadipate semialdehyde is then oxidized into $\alpha$-aminoadipate by the enzyme AASADH using $\mathrm{NAD}(\mathrm{P})^{+}$as cofactors.

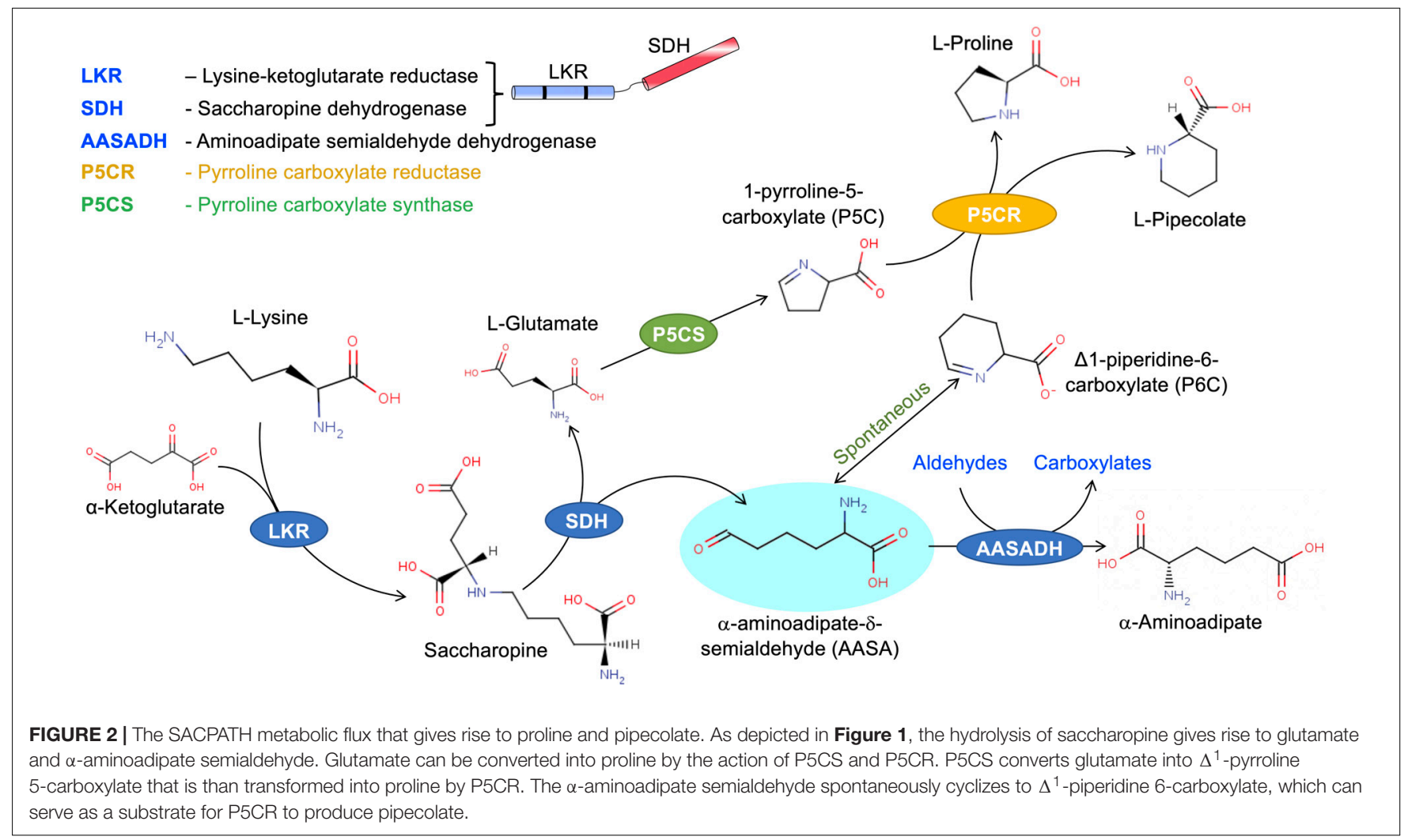

use an array of aldehydes as substrates (Brocker et al., 2010; Kyiota et al., 2015) but seems to be the only ALD capable of using $\alpha$-aminoadipate semialdehyde as a substrate, as null mutations of this enzyme in humans cause the disease pyridoxine-dependent epilepsy (PDE) (Mills et al., 2006). The ability of AASADH to use multiple aldehydes as substrates, such as betaine aldehyde, hexanal, octanal, non-anal, trans-2-non-anal, benzaldehyde and propanal (Brocker et al., 2010; Kyiota et al., 2015), makes it a scavenger of aldehydes generated by lipid peroxidation, carbohydrate metabolism, amino acid catabolism, and oxidative 
stress, among others. AASADH has gained attention for its roles in the abiotic stress response and its involvement in human PDE (Mills et al., 2006; Rodrigues et al., 2006; Brocker et al., 2010, 2011; Kyiota et al., 2015; Crowther et al., 2019; Yang et al., 2019).

A detailed characterization of an AASADH enzyme was performed in a study of its role in hyperosmotic protection and cellular homeostasis in mouse (Brocker et al., 2010). AASADH is encoded by a single gene that generates differentially spliced transcripts producing polypeptides targeted to the cytosol and to the mitochondria. The transcript encoding the mitochondrialtargeted AASADH uses an alternative start codon that adds a mitochondrial signal sequence to the amino-terminus of the protein (Brocker et al., 2010).

Interestingly, compared with that for $\alpha$-aminoadipate semialdehyde, the $\mathrm{Km}$ for AASADH from both animals and maize was shown to be 4-20-fold lower for non-anal, hexanal, octanal, benzaldehyde, and propanal (Brocker et al., 2010; Kyiota et al., 2015). The much higher affinity for the aliphatic aldehydes suggests an essential role of AASADH in alleviating the cellular toxicity of aldehydes, especially when plants are subjected to stress.

\section{P5CS}

P5CS is a bifunctional polypeptide containing $\gamma$-glutamyl kinase (GK) and glutamate-5-semialdehyde dehydrogenase (GPR) domains (Zhang et al., 1995; Perez-Arellano et al., 2010; Verslues and Sharma, 2010). In plants, there are two isoforms, P5CS1 and P5CS2, which are encoded by two closely related genes (Szekely et al., 2008). P5CS1 localizes in chloroplasts, whereas P5CS2 localizes to the cytosol. The P5CS amino acid sequence is highly conserved across species, which suggests its general biological relevance. The GPR catalytic domain converts $\gamma$-glutamyl phosphate to glutamate5-semialdehyde, which then spontaneously cyclizes into $\Delta^{1}$ pyrroline-5-carboxylate (Verslues and Sharma, 2010) (Figure 2). P5CS is a rate-limiting enzyme and thus is critical in increasing proline concentrations when plants are subjected to abiotic stress (Szabados and Savoure, 2009; Verslues and Sharma, 2010; Bhaskara et al., 2015). GK activity, but not GPR activity, is subject to feedback inhibition by proline, implying that the level of free amino acids in cellular medium feedback regulates enzyme activity (Zhang et al., 1995).

\section{P5CR}

P5CR has been less studied in plants. The enzyme converts $\Delta^{1}$ pyrroline-5-carboxylate into proline (Figure 2); it is ubiquitous to most plant organs, and its expression is particularly high in soybean root nodules (Szoke et al., 1992). The enzyme is encoded by a single gene and has a $29 \mathrm{kDa}$ monomeric native form. P5CR is mostly localized in the cytosol, although a small proportion of the enzyme also localizes in plastids (Szoke et al., 1992). Despite being encoded by a single gene, two isoforms of the enzymes P5CR-1 and P5CR-2 have been identified in spinach: P5CR-2 is a plastid isoform (Verslues and Sharma, 2010). P5CR is not rate limiting for the synthesis of proline and its expression seems not to be induced by abiotic stress (Verslues and Sharma, 2010), although upregulation of expression of the enzyme has been shown in plants and bacteria subjected to osmotic/salt stress (Neshich et al., 2013; Koenigshofer and Loeppert, 2019).

\section{METABOLIC FLUX THROUGH THE SACPATH}

In higher eukaryotes, the metabolic flux from lysine to $\alpha$-aminoadipate (Figure 2) is unidirectional, since there is no evidence of the involvement of the SACPATH in lysine biosynthesis. In certain bacteria, lysine is directly oxidized into $\alpha$-aminoadipate semialdehyde by the enzyme lysine dehydrogenase (Neshich et al., 2013). However, $\alpha$-aminoadipate semialdehyde is highly toxic to eukaryotic cells. In humans, for example, its accumulation causes PDE (Mills et al., 2006). Thus, $\alpha$-aminoadipate semialdehyde must be maintained at low concentrations by AASADH and P5CR to produce $\alpha$-aminoadipate and pipecolate, respectively (Figure 2). Metabolic flux toward $\alpha$-aminoadipate and pipecolate occurs when plants are exogenously fed lysine (Moulin et al., 2006; Kyiota et al., 2015). The glutamate formed by saccharopine hydrolysis can be used for the synthesis of proline (Figure 2). Proline synthesis from lysine was first shown by $\mathrm{C}^{14}$-lysine feeding in the immature endosperm of wheat, maize, and barley (Lawrence and Grant, 1964; Sodek and Wilson, 1970; Brandt, 1975). Although LKR/SDH enzymes were unknown at that time, the SACPATH must have been responsible for the conversion of lysine to proline.

The conversion of $\Delta^{1}$-piperideine-6-carboxylate to pipecolate (Figure 2) was demonstrated in animals in a study designed to investigate whether fibroblasts catabolize lysine through the SACPATH or through the pipecolate pathway, both of which converges to $\alpha$-aminoadipate semialdehyde (Struys and Jakobs, 2010). A human AASADH-deficient fibroblast cell line fed either $\mathrm{L}-\left[\alpha_{-}{ }^{15} \mathrm{~N}\right]$ or $\mathrm{L}-\left[\varepsilon_{-}{ }^{15} \mathrm{~N}\right]$ lysine catabolized $\mathrm{L}-\left[\alpha_{-}{ }^{15} \mathrm{~N}\right]$ lysine into $\left[\alpha-{ }^{15} \mathrm{~N}\right]$ saccharopine, $\left[\alpha-{ }^{15} \mathrm{~N}\right]$ aminoadipic semialdehyde, $[\alpha-$ $\left.{ }^{15} \mathrm{~N}\right] \Delta^{1}$-pyrroline-6-carboxylate, and $\left[\alpha-{ }^{15} \mathrm{~N}\right]$ pipecolate (Struys and Jakobs, 2010). The incubation of recombinant human P5CR with $\Delta^{1}$-piperideine-6-carboxylate further demonstrated its enzymatic conversion solely into pipecolate (Struys et al., 2014). Previously, studies in an animal model argued that the pipecolate pathway is preferred for lysine catabolism in the brain, while the SACPATH is preferred for the liver and kidney (Sauer et al., 2011; Hallen et al., 2013; Posset et al., 2014). An accurate experimental design to follow the time course of lysine degradation in the mouse liver, kidney, and brain revealed that the catabolic activity peak in response to exogenous lysine fed to the animals occurs $2 \mathrm{~h}$ after intraperitoneal injection of lysine (Pena et al., 2016). By the use of this experimental setup, intraperitoneal injection of L- $\left[\alpha-{ }^{15} \mathrm{~N}\right]$ or $\mathrm{L}-\left[\varepsilon_{-}{ }^{15} \mathrm{~N}\right]$ lysine revealed that lysine catabolism in the mouse liver, kidney, and brain occurs through the SACPATH (Pena et al., 2017). There was no evidence for the synthesis of $\alpha$-aminoadipate from the pipecolate pathway. In addition, pipecolate derived from L- $\left[\alpha-{ }^{15} \mathrm{~N}\right]$ lysine confirmed its synthesis through the SACPATH in the liver, kidney, and brain (Pena et al., 2017). The prevalence of the SACPATH for lysine catabolism and pipecolate synthesis was also recently observed 
for human neuronal progenitor cells and fibroblasts. Isotopic tracing experiments, similar to those carried out in mice, revealed that lysine catabolism occurs exclusively through the SACPATH (Crowther et al., 2019). L- $\left[\alpha_{-}{ }^{15} \mathrm{~N}\right]$ or $\mathrm{L}-\left[\varepsilon^{-}{ }^{15} \mathrm{~N}\right] l y$ sine feeding experiments in bean also indicate preferred incorporation of the $\alpha-\mathrm{N}$ group into pipecolate (Schutte and Seeling, 1967 was coted in the text as cited by Hartmann et al., 2017).

\section{INVOLVEMENT OF THE SACPATH IN THE ABIOTIC AND BIOTIC STRESS RESPONSE}

Lysine catabolism through the SACPATH is highly responsive to abiotic stress (Moulin et al., 2006; Boex-Fontvieille et al., 2013; Kyiota et al., 2015; Michaletti et al., 2018; Wu et al., 2018; Dudziak et al., 2019; Yadav et al., 2019; You et al., 2019). Nevertheless, to date, there is no direct evidence for the involvement of the SACPATH in the biotic stress response. Instead, the recent discovery of the NHP pathway of lysine catabolism has shown its critical role for the induction of SAR (for a review, see Hartmann and Zeier, 2018).

In the previous sections, we presented the current knowledge of the enzymatic properties that support the synthesis through the SACPATH of two of the most critical osmolytes, proline and pipecolate (Szabados and Savoure, 2009; Bhaskara et al., 2015; Pérez-García et al., 2019). However, the question is how the SACPATH enzymatic activities and derived metabolites contribute to abiotic and biotic stress responses. The response of the SACPATH to osmotic, drought and salt stress is conserved in plants, animals, and bacteria, indicating its evolutionary importance to cope with abiotic stress. Notably, the enzymes LKR/SDH and AASADH are co-upregulated at the transcriptional level by exogenously applied lysine in plants, animals, and bacteria (Papes et al., 1999; Stepansky et al., 2005; Neshich et al., 2013; Kyiota et al., 2015). The induction of LKR activity by phosphorylation in a lysine-dependent manner implies that this enzyme is quickly activated to produce saccharopine once lysine levels start rising. The immediate increase in LKR activity stimulates increases in SDH activity, as the two activities occur within the same polypeptide. The immediate consequence of these two reaction steps is the increase in the concentration of $\alpha$-aminoadipate semialdehyde, which would require an increase in AASADH and perhaps P5CR activities to maintain $\alpha$-aminoadipate semialdehyde concentrations below toxic levels.

Metabolic perturbations such as those imposed by osmotic, drought and salt stress, will lead to the recycling of cellular catabolic processes, including proteomic hydrolysis (for a review, see Hildebrandt et al., 2015). Stress-induced protein hydrolysis results in increased free lysine levels. Increased lysine pool could also result from the induction of the aspartate (AK) pathway for lysine biosynthesis (Figure 1A) that has also been shown to be induced under abiotic stress (Pratelli and Pilot, 2014). However, the AK pathway for lysine biosynthesis localizes in the chloroplast and the amino acid needs to be transported to the cytosol and other organelles to be effectively metabolized.
One of the most well-characterized amino acid transporters that operate in the chloroplast is the LHT1, which transport lysine and histidine (Fischer et al., 1998; Pratelli and Pilot, 2014). LHT1 has also been shown to be induced under abiotic stress and thus connect lysine biosynthesis to its catabolism in an integrated stress response metabolic network (Pratelli and Pilot, 2014). Increased free lysine levels have been observed in plants subjected to abiotic stress, but of relevance is the observation of higher levels of free lysine in sesame and wheat drought-tolerant genotypes compared with susceptible genotypes subjected to drought stress (Yadav et al., 2019; You et al., 2019). These observations suggest that abiotic stress-tolerant genotypes may have selected traits associated with the upregulation of the SACPATH. Indeed, transcriptomic, proteomic and metabolomic profiling of abiotic stress-tolerant sesame and wheat genotypes have revealed an induction of metabolic flux throughout the SACPATH to a greater extent in drought-tolerant genotypes than in drought-susceptible genotypes, which resulted in the accumulation of saccharopine and $\alpha$-aminoadipate (Michaletti et al., 2018; You et al., 2019).

The role of the SACPATH in the osmotic stress response was first reported in rapeseed leaf disks treated with polyethylene glycol solutions at -1 to $-4 \mathrm{MPa}$ (Moulin et al., 2006). It was found that hyperosmotic treatment of rapeseed leaf disks induces an increase in LKR/SDH transcript abundance and enzymatic activity, which correlates with decreased levels of free lysine and increased levels of pipecolate (Moulin et al., 2006). Furthermore, the LKR/SDH activity and pipecolate concentration decrease with the return of the leaf disks to hypoosmotic conditions (Moulin et al., 2006). Exogenous administration of lysine induces LKR/SDH activity and increases pipecolate levels to the same extent as does hyperosmotic treatment, which supports the hypothesis that pipecolate is synthesized via the SACPATH (Moulin et al., 2006). Other abiotic stresses, such as those caused by different illumination conditions, also induce the SACPATH. A metabolic genome-wide association study of 309 Arabidopsis accessions subjected to light and dark treatments revealed a strong stress association between saccharopine accumulation and single-nucleotide polymorphisms (SNPs) at LKR/SDH loci. Saccharopine accumulates under low-light or dark conditions, indicating that the induction of the SACPATH is essential under these stressful conditions (Wu et al., 2018). Moreover, the osmolytes proline and pipecolate accumulate to high levels in halophytes, indicating their role in adaptative metabolism under stressful saline conditions (Slama et al., 2015; Jawahar et al., 2019).

The lysine-to- $\alpha$-aminoadipate metabolic flux requires the coordinated activities of $\mathrm{LKR} / \mathrm{SDH}$ and AASADH. Indeed, $\mathrm{LKR} / \mathrm{SDH}$, and AASADH are co-upregulated at transcriptional and translational levels in response to exogenous administration of lysine in plants, animals, and bacteria (Neshich et al., 2013; Kyiota et al., 2015; Pena et al., 2016, 2017). The synthesis of pipecolate from $\Delta^{1}$-piperideine-6-carboxylate by the enzyme P5CR (Struys and Jakobs, 2010; Struys et al., 2014; Pena et al., 2017) suggest that the SACPATH contributes to abiotic stress alleviation by increasing cellular concentrations of both proline and pipecolate (Figure 2). However, the accumulation of saccharopine when plants are subjected to abiotic stress 


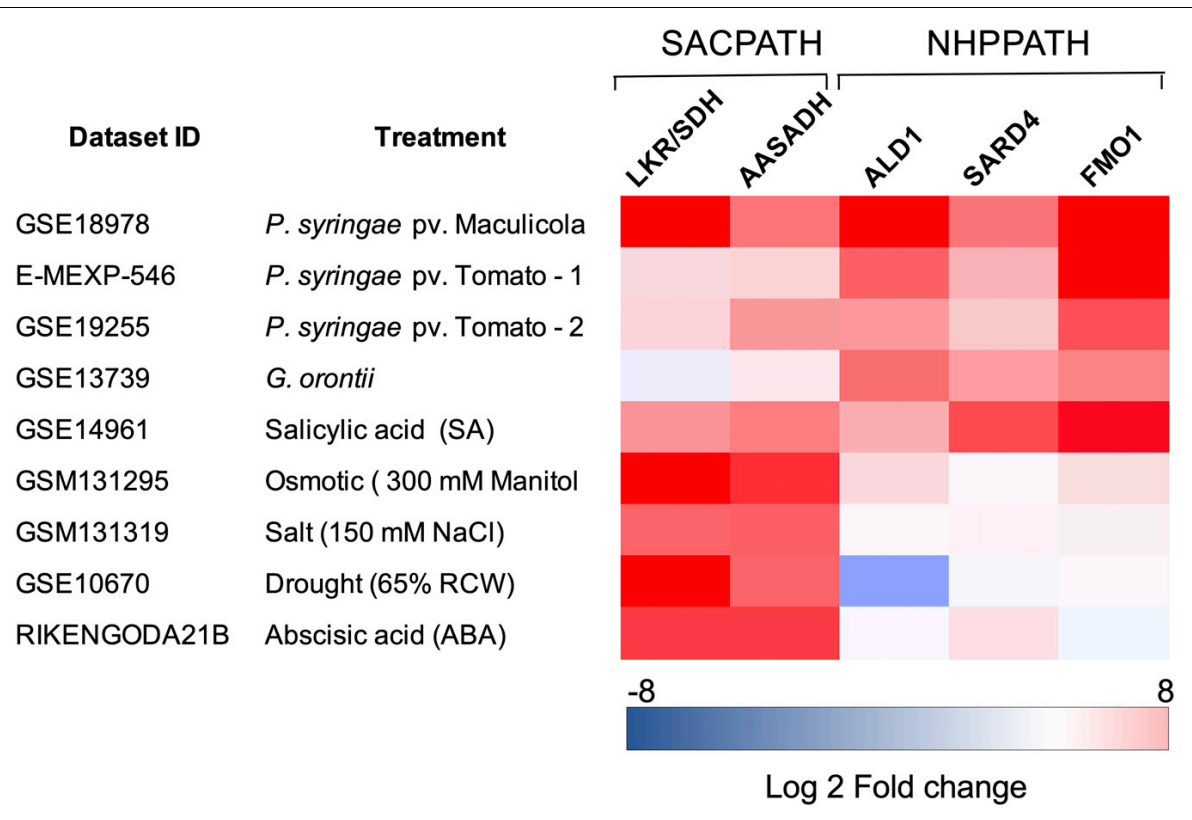

FIGURE 3 | Expression profiling of the core genes of the SACPATH and NHP pathway for lysine catabolism in Arabidopsis subjected to biotic and abiotic stresses. Expression profiling datasets of the genes encoding LKR/SDH and AASADH of the SACPATH and ALD1, SARD4, and FMO1 of the NHP pathway, were retrieved from public databases and analyzed using the Genevestigator platform ${ }^{1}$. Only the data of wild type (Col-0 or Ws-0) subjected to control and stress treatments were used in this analysis. The following datasets were used: GSE18978, leaves inoculated or not inoculated with Pseudomonas syringae ES4326; E-MEXP-546, leaves inoculated or not inoculated with the $P$. syringae pv. tomato avirulent strain DC3000 avrRpm1; GSE19255; leaves inoculated or not inoculated with $P$, syringae pv. tomato; GSE13739, leaves inoculated or not inoculated with the G. orontii MGH isolate; GSE14961, seedlings grown for 1 day in 2 mM salicylic acid; GSM131295, 16-day-old seedlings grown in 300 mM mannitol (osmotic stress); GSM131319, 16-day-old seedlings grown in 150 mM NaCl (salt stress); GSE10670, plants subjected to drought stress; and RIKENGODA21B, seedlings treated with $10 \mu \mathrm{M}$ ABA. The values are expressed as log2 of the fold change between treated and untreated plants.

(Michaletti et al., 2018; You et al., 2019) must be further investigated. In animals, the SACPATH is exclusively located in the mitochondria (Blemings et al., 1994), an organelle essential for sustaining cellular energy homeostasis under stress conditions. However, the accumulation of saccharopine is toxic to mitochondria (Zhou et al., 2018). In humans, mutations in the LKR domain of the LKR/SDH enzyme lead to lysinuria, an asymptomatic disease (Sacksteder et al., 2000), but mutations in the SDH domain lead to saccharopinuria, a disease associated with severe brain malfunction and retardation (Houten et al., 2013). Saccharopinuria leads to the accumulation of high levels of saccharopine, which induces mitochondrial malfunction that can be reversed by the expression of a wild-type mitochondrial-targeted SDH (Zhou et al., 2018). In Arabidopsis, a monofunctional SDH probably produced from the same gene encoding the bifunctional enzyme localizes in the mitochondria (Fuchs et al., 2019). Under stress conditions, the targeting of $\mathrm{SDH}$ to the mitochondria may help reduce the concentration of saccharopine inside the organelle and maintain its normal function. Another point of significance of the SACPATH when plants are subjected to abiotic stress is the synthesis $\alpha$-aminoadipate semialdehyde. $\alpha$-Aminoadipate semialdehyde should be maintained at a low level because it is highly reactive; can bind to proteins, nucleic acids, and small

${ }^{1}$ https://genevestigator.com/gv/ molecules; and can interfere with an array of cellular processes. It is possible that $\alpha$-aminoadipate semialdehyde acts in the upregulation of AASADH (Brocker et al., 2010, 2011; Kyiota et al., 2015) to help rid cells of aldehydes generated from stressinduced metabolic processes (Singh et al., 2012). Additionally, AASADH can produce other osmolytes such as betaine from betaine aldehyde that would help alleviate stress imposed by salt, osmotic, and drought (Singh et al., 2012) and also sustain cellular energy homeostasis (Yang et al., 2019).

Another relevant question is whether the pipecolate generated by the SACPATH contributes to the stimulation of the plant immune response in addition to pipecolate produced through the NHP pathway (Hartmann et al., 2017, 2018; Chena et al., 2018; Hartmann and Zeier, 2018; Wang C. et al., 2018; Wang Y. et al., 2018). Pipecolate accumulates to high levels upon pathogen attack, stimulating the synthesis of NHP, which subsequently activates SAR at the infected site and in distant leaves (Hartmann and Zeier, 2018). Engineering the NHP pathway by the transient overexpression of ALD1 and FMO1 has been shown to activate the SAR response in tomato plants (Holmes et al., 2019). Is the pipecolate produced by the NHP pathway the only pipecolate source for SAR activation or can the pipecolate produced by the SACPATH contribute, at least in part, to immune response? Perhaps the different intracellular localization of the SACPATH and the NHP pathway may have a role in differentiating the pipecolate source in the activation of the biotic and abiotic 


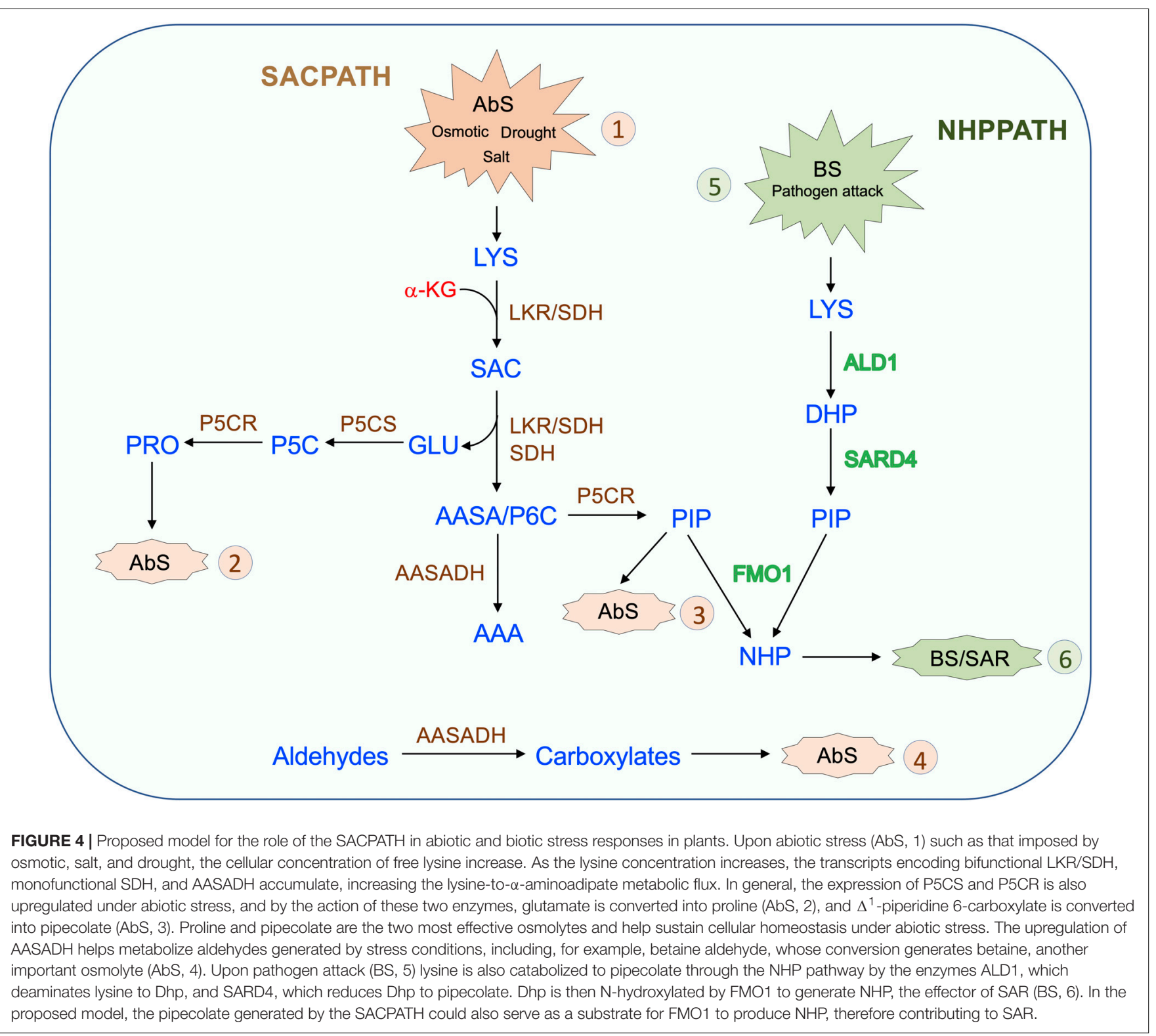

stress response. The bifunctional LKR/SDH, at least in immature maize endosperm, is located in the cytosol, but a monofunctional $\mathrm{SDH}$ has been found that targets the mitochondria. AASADH is localized both in the cytosol and in the mitochondria but not in the plastids. The intracellular localization of P5CR is not well established, but it has also been found in plastids. The NHP pathway seems to be localized in the chloroplasts of developed leaves. Thus, it is possible that the pipecolate produced from the SACPATH and NHP is differentially compartmentalized so that the NHP pathway, whose enzymes are located in the chloroplast, is directly associated with the biotic stress response and therefore is preferentially associated with pathogen infection. We took advantage of public datasets designed to study the transcriptomic profiling of Arabidopsis to interrogate whether the SACPATH and NHP pathway are differentially expressed under biotic and abiotic stress. A heatmap was constructed to profile the transcriptional response of LKR/SDH, AASADH, ALD1, SARD4, and FMO1 in Arabidopsis subjected to biotic and abiotic stress conditions (Figure 3). Although the datasets were retrieved from distinct experiments, we can clearly observe that the expression of the genes encoding the enzymes of the SACPATH and the NHP pathway are co-upregulated under biotic stress imposed by infection with $P$. syringae or treatment with salicylic acid (Figure 3). In the case of infection with the fungus Golovinomyces orontii, only the NHP pathway is upregulated. In contrast, under abiotic stress imposed by osmotic, salt, or drought stress or when plants were treated with abscisic acid (ABA), the SACPATH was preferentially upregulated (Figure 3 ). This means that the SACPATH may contribute to the synthesis of NHP from the pipecolate produced from $\Delta^{1}$-pyrroline-6-carboxylate through the enzymatic activity of P5CR (Figure 3) but the NHP pathway may not contribute with abiotic stress response. 


\section{CONCLUSION}

The SACPATH for lysine catabolism is widespread across kingdoms, except for fungi, which use the pathway for lysine synthesis. Upon intracellular increase in lysine concentration, due to metabolism-related organ development programs or responses to abiotic and biotic stress, LKR undergoes posttranscriptional modification mediated by $\mathrm{Ca}^{2+}$ and phosphorylation to initiate SACPATH reactions, first by LKR activity to produce saccharopine and then by $\mathrm{SDH}$ activity to produce $\alpha$-aminoadipate semialdehyde and glutamate (Figure 4, Abs 1). Increased intracellular free lysine also induces the transcriptional upregulation of the $L K R / S D H$ and $A A S A D H$ genes which boost the lysine-to- $\alpha$-aminoadipate metabolic flux and the use of glutamate to produce proline (Figure 4, Abs 2) and the intermediary $\mathrm{D}^{1}$-piperideine-6-carboxylate to produce pipecolate (Figure 4, Abs 3). A monofunctional SDH enzyme is targeted to the mitochondria, perhaps to help maintain saccharopine levels below toxic levels. The upregulation of AASADH in turn would help to maintain $\alpha$-aminoadipate semialdehyde at low levels and help to reduce the levels of the stress-generated aldehydes, thus contributing to the alleviation of the toxic effects on cellular metabolism (Figure 4, Abs 4). The proline and pipecolate generated by the SACPATH must contribute to alleviating the cellular impact of osmotic, salt, and drought stress (Figure 4, Abs 1). However, the cellular availability of pipecolate generated by the SACPATH could also contribute to the production of NHP and thus induce SAR, as the enzymes LKR/SDH and AASADH are induced by pathogen attack (Figure 3). Perhaps the main difference between the biotic and abiotic stress responses associated with lysine catabolism through the SACPATH and the NHP pathway resides in the mode of action of these two conditions. Pathogen infection is punctual (Figure 4, BS 5), and, independent of the plant organ, the infection spots comprise a limited number of cells. At the infection site, lysine catabolism through the

\section{REFERENCES}

Anderson, O. D., Coleman-Derr, D., Gu, Y. Q., and Heath, S. (2010). Structural and transcriptional analysis of plant genes encoding the bifunctional lysine ketoglutarate reductase saccharopine dehydrogenase enzyme. BMC Plant Biol. 10:113. doi: 10.1186/1471-2229-10-113

Arruda, P., Kemper, E. L., Papes, F., and Leite, A. (2000). Regulation of lysine catabolism in higher plants. Trends Plant Sci. 5, 324-330. doi: 10.1016/s13601385(00)01688-1685

Arruda, P., and Neshich, I. P. (2012). Nutritional-rich and stress-tolerant crops by saccharopine pathway manipulation. Food Energy Secur. 1, 141-147. doi: $10.1002 /$ fes 3.9

Arruda, P., Sodek, L., and da Silva, W. J. (1982). Lysine-ketoglutarate reductase activity in developing maize endosperm. Plant Physiol. 69, 988-989. doi: 10. 1104/pp.69.4.988

Azevedo, R. A., Damerval, C., Landry, J., Lea, P. J., Bellato, C. M., Meinhardt, L. W., et al. (2003). Regulation of maize lysine metabolism and endosperm protein synthesis by opaque and floury mutations. Eur. J. Biochem. 270, 4898-4908. doi: 10.1111/j.1432-1033.2003.03890.x

Azevedo, R. A., and Lea, P. J. (2001). Lysine metabolism in higher plants. Amino Acids 20, 261-279. doi: 10.1007/s007260170043
NHP pathway must be intense, pipecolate accumulates at high levels, and NHP is produced and spread over long distances to mediate the SAR response (Figure 4, BS 6). However, a prerequisite to start the process is the rise of free lysine concentration in the infection site. The rise of free lysine concentrations independent, whether it is due to biotic or abiotic stress, induces the SACPATH (Figure 3) that can also mediate the synthesis of NHP. In contrast, osmotic, drought, and salt stress affect whole plant organs simultaneously, leading to the activation of the SACPATH and accumulation of pipecolate. Since abiotic stress must debilitate the plant, making it more prone to pathogen attack, the pipecolate produced by the SACPATH may help to stimulate the immune response in these circumstances.

\section{AUTHOR CONTRIBUTIONS}

PA organized and wrote the manuscript. PB contributed with the expression profiling analysis of SACPATH and NHP pathways.

\section{FUNDING}

This work was supported by a grant from FAPESP to PA 2016/23218-0. PB received a FAPESP postdoc fellowship 14/17634-5.

\section{ACKNOWLEDGMENTS}

The authors acknowledge The State University of Campinas (UNICAMP), The Brazilian Agricultural Research Corporation (EMBRAPA) and The São Paulo Research Foundation (FAPESP) for housing and funding the Genomics for Climate Changes Research Center (GCCRC).
Bhaskara, G. B., Yang, T.-H., and Verslues, P. E. (2015). Dynamic proline metabolism: importance and regulation in water limited environments. Front. Plant Sci. 6:484. doi: 10.3389/fpls.2015.00484

Blemings, K. P., Crenshaw, T. D., Swick, R. W., and Benevenga, N. J. (1994). Lysine- $\alpha$-ketoglutarate reductase and saccharopine dehydrogenase are located only in the mitochondrial matrix in rat liver. J. Nutr. 124, 1215-1221. doi: $10.1093 / \mathrm{jn} / 124.8 .1215$

Boex-Fontvieille, E. R. A., Gauthier, P. P. G., Gilard, F., Hodges, M., and Tcherkez, G. G. B. (2013). A new anaplerotic respiratory pathway involving lysine biosynthesis in isocitrate dehydrogenase-deficient Arabidopsis mutants. New Phytol. 199, 673-682. doi: 10.1111/nph.12319

Brandt, A. B. (1975). In vivo incorporation of [14C] lysine into the endosperm proteins of wild type and high-lysine barley. FEBS Lett. 52, 288-291. doi: 10. 1016/0014-5793(75)80827-1

Brochetto-Braga, M. R., Leite, A., and Arruda, P. (1992). Partial purification and characterization of lysine-ketoglutarate reductase in normal and opaque-2 maize endosperms. Plant Physiol. 98, 1139-1147. doi: 10.1104/pp.98.3.1139

Brocker, C., Cantore, M., Failli, P., and Vasiliou, V. (2011). Aldehyde dehydrogenase 7A1 (ALDH7A1) attenuates reactive aldehyde and oxidative stress induced cytotoxicity. Chem. Biol. Interact 191, 269-277. doi: 10.1016/j. cbi.2011.02.016 
Brocker, C., Lassen, N., Estey, T., Pappa, A., Cantore, M., Orlova, V. V., et al. (2010). Aldehyde dehydrogenase 7A1 (ALDH7A1) Is a novel enzyme involved in cellular defense against hyperosmotic stress. J. Biol. Chem. 285, 18452-18463. doi: 10.1074/jbc.M109.077925

Bunsupa, S., Katayama, K., Ikeura, E., Oikawa, A., Toyooka, K., Saito, K., et al. (2012). Lysine decarboxylase catalyzes the first step of quinolizidine alkaloid biosynthesis and coevolved with alkaloid production in leguminosae. Plant Cell 24, 1202-1216. doi: 10.1105/tpc.112.095885

Chena, Y. C., Holmes, E. C., Rajniak, J., Kima, J. G., Tang, S., Fischer, C. R., et al. (2018). N-hydroxy-pipecolic acid is a mobile metabolite that induces systemic disease resistance in Arabidopsis. Proc. Natl. Acad. Sci. U.S.A. 115, e4920-e4929. doi: $10.1073 /$ pnas. 1805291115

Crowther, L. M., Mathis, D., Poms, M., and Plecko, B. (2019). New insights into human lysine degradation pathways with relevance to pyridoxine-dependent epilepsy due to antiquitin deficiency. J. Inherit Metab. Dis. 42, 620-628. doi: 10.1002/jimd.12076

Dudziak, K., Zapalska, M., Börner, A. M., Szczerba, H., Kowalczyk, K., and Nowak, M. (2019). Analysis of wheat gene expression related to the oxidative stress response and signal transduction under short-term osmotic stress. Sci. Rep. 9:2743. doi: 10.1038/s41598-019-39154-w

Fischer, W.-N., Bruno, A., Rentsch, D., Krolkiewicz, S., Tegeder, M., Breitkreuz, K., et al. (1998). Amino acid transport in plants. Trends Sci. 3:188. doi: 10.1016/ S1360-1385(98)01231-X

Fuchs, P., Rugen, N., Carrie, C., Elsasser, M., Finkemeier, I., Giese, J., et al. (2019). Single organelle function and organization as estimated from Arabidopsis mitochondrial proteomics. Plant J. 101, 420-441. doi: 10.1111/tpj.14534

Gaziola, S. A., Sodek, L., Arruda, P., Lea, P. J., and Azevedo, R. A. (2000). Degradation of lysine in rice seeds: effect of calcium, ionic strength, S-adenosylmethionine and S-2-aminoethyl-L-cysteine on the lysine 2-oxoglutarate reductase-saccharopine dehydrogenase bifunctional enzyme. Physiol. Plant. 110, 164-171. doi: 10.1034/j.1399-3054.2000. 110204.x

Gaziola, S. A., Teixeira, C. M. G., Lugli, L., Sodek, L., and Azevedo, R. A. (1997). The enzymology of lysine catabolism in rice seeds Isolation, characterization, and regulatory properties of a lysine 2-oxoglutarate reductase/saccharopine dehydrogenase bifunctional polypeptide. Eur. J. Biochem. 247, 364-371. doi: 10.1111/j.1432-1033.1997.00364.x

Gonçalves-Butruille, M., Szajner, P., Torigoi, E., Leite, A., and Arruda, P. (1996). Purification and characterization of the bifunctional enzyme lysineketoglutarate reductase-saccharopine dehydrogenase from maize. Plant Physiol. 110, 765-771. doi: 10.1104/pp.110.3.765

Hallen, A., Jamie, J. F., and Cooper, A. J. L. (2013). Lysine metabolism in mammalian brain: an update on the importance of recent discoveries. Amino Acids 45, 1249-1272. doi: 10.1007/s00726-013-1590-1591

Hartmann, M., Kim, D., Bernsdorff, F., Ajami-Rashidi, Z., Scholten, N., Schreiber, S., et al. (2017). Biochemical principles and functional aspects of pipecolic acid biosynthesis in plant immunity. Plant Physiol. 174, 124-153. doi: 10.1104/pp. 17.00222

Hartmann, M., and Zeier, J. (2018). L-lysine metabolism to N-hydroxypipecolic acid: an integral immune-activating pathway in plants. Plant J. 96, 5-21. doi: 10.1111/tpj.14037

Hartmann, M., Zeier, T., Bernsdorff, F., Reichel-Deland, V., Kim, D., Hohmann, M., et al. (2018). Flavin monooxygenase-generated N-hydroxypipecolic acid is a critical element of plant systemic immunity. Cell 173, 456-469. doi: 10.1016/j. cell.2018.02.049

Hildebrandt, T. M., Nunes Nesi, A., Araujo, W. L., and Braun, H. P. (2015). Amino acid catabolism in plants. Mol. Plant 8, 1563-1579. doi: 10.1016/j.molp.2015.09. 005

Holmes, E. C., Chen, Y.-C., Sattely, E. S., and Mudgett, M. B. (2019). An engineered pathway for N-hydroxy-pipecolic acid synthesis enhances systemic acquired resistance in tomato. Sci. Signal. 12:eaay3066. doi: 10.1126/scisignal.aay3066

Houten, S. M., Te Brinke, T., Denis, S., Ruiter, J. P., Knegt, A. C., de Klerk, J. B., et al. (2013). Genetic basis of hyperlysinemia. Orphanet J. Rare Dis. 8:57.

Jawahar, G., Rajasheker, G., Maheshwari, P., Punita, D. L., Jalaja, N., Kumari, P. H., et al. (2019). Osmolyte diversity, distribution, and their biosynthetic pathways. Plant Signal. Mol. 28, 449-458. doi: 10.1016/B978-0-12-816451-8.00028-29

Kemper, E. L., Cord-Neto, G., Capella, A. N., Gonçalves-Butruile, M., Azevedo, R. A., and Arruda, P. (1998). Structure and regulation of the bifunctional enzyme lysine-oxoglutarate reductase-saccharopine dehydrogenase in maize. Eur. J. Biochem. 253, 720-729. doi: 10.1046/j.1432-1327.1998.2530720.x

Kemper, E. L., Cord-Neto, G., Papes, F., Moraes, K. C. M., Leite, A., and Arruda, P. (1999). The role of opaque 2 in the control of lysine-degrading activities in developing maize endosperm. Plant Cell 11, 1981-1993. doi: 10.1105/tpc.11. 10.1981

Koenigshofer, H., and Loeppert, H. G. (2019). The up-regulation of proline synthesis in the meristematic tissues of wheat seedlings upon short-term exposure to osmotic stress. J. Plant Physiol. 237, 21-29. doi: 10.1016/j.jplph. 2019.03.010

Kyiota, E., Pena, I. A., and Arruda, P. (2015). The saccharopine pathway in seed development and stress response of maize. Plant Cell Environ. 38, 2450-2461. doi: $10.1111 /$ pce. 12563

Lawrence, J. M., and Grant, D. R. (1964). Incorporation of lysine-C14 into the developing grain of wheat. Arch. Biochem. Biophys. 104, 73-78. doi: 10.1016/ S0003-9861(64)80036-80039

Lobell, D. B., Schlenker, W., and Costa-Roberts, J. (2011). Climate trends and global crop production since 1980. Science 333, 616-620. doi: 10.1126/science. 1204531

Long, X., Liu, Q., Chan, M., Wang, Q., and Sun, S. S. M. (2013). Metabolic engineering and profiling of rice with increased lysine. Plant Biotech. J. 11:490. doi: $10.1111 /$ pbi.12037

Markovitzs, P. J., and Chuang, D. T. (1987). The bifunctional aminoadipic semialdehyde synthase in lysine degradation. J. Biol. Chem. 262, 9353-9358.

Michaletti, A., Naghavi, M. R., Toorchi, M., Zolla, L., and Rinalducci, S. (2018). Metabolomics and proteomics reveal drought-stress responses of leaf tissues from spring-wheat. Sci. Rep. 8:5710. doi: 10.1038/s41598-018-24012-y

Mills, P. B., Struys, E., Jakobs, V., Plecko, B., Baxter, P., Baumgartner, M., et al. (2006). Mutations in antiquitin in individuals with pyridoxine-dependent seizures. Nat. Med. 12, 307-309. doi: 10.1038/nm1366

Moulin, M., Deleu, C., Larher, F., and Bouchereau, A. (2006). The lysineketoglutarate reductase-saccharopine dehydrogenase is involved in the osmoinduced synthesis of pipecolic acid in rapeseed leaf tissues. Plant Physiol. Biochem. 44, 474-482. doi: 10.1016/j.plaphy.2006.08.005

Neshich, I. A. P., Kiyota, E., and Arruda, P. (2013). Genome-wide analysis of lysine catabolism in bacteria reveals new connections with osmotic stress resistance. ISME J. 7, 2400-2410. doi: 10.1038/ismej.2013.123

Papes, F., Kemper, E. L., Cord-Neto, G., Langone, F., and Arruda, P. (1999). Lysine degradation through the saccharopine pathway in mammals: involvement of both bifunctional and monofunctional lysine-degrading enzymes in mouse. Biochem. J. 344, 555-563.

Pena, I. A., Marques, L. A., Laranjeira, A. B. A., Yunes, J. A., Eberlin, M. N., and Arruda, P. (2016). Simultaneous detection of lysine metabolites by a single LCMS/MS method: monitoring lysine degradation in mouse plasma. Springerplus 5:172. doi: 10.1186/s40064-016-1809-1801

Pena, I. A., Marques, L. A., Laranjeira, A. B. A., Yunes, J. A., Eberlin, M. N., MacKenzie, A., et al. (2017). Mouse lysine catabolism to aminoadipate occurs primarily through the saccharopine pathway; implications for pyridoxine dependent epilepsy (PDE). Biochim. Biophys. Acta Mol. Basis Dis. 1863, 121128. doi: 10.1016/j.bbadis.2016.09.006

Perez-Arellano, I., Carmona-Alvarez, F., Martinez, A. I., Rodriguez-Diaz, J., and Cervera, J. (2010). Pyrroline-5-carboxylate synthase and proline biosynthesis: from osmotolerance to rare metabolic disease. Protein Sci. 19, 372-382. doi: $10.1002 /$ pro. 340

Pérez-García, F., Brito, L. F., and Wendisch, W. F. (2019). Function of L-pipecolic acid as compatible solute in Corynebacterium glutamicum as basis for its production under hyperosmolar conditions. Front. Microbiol. 10:340. doi: 10. 3389/fmicb. 2019.00340

Pompeu, G. B., Vendemiatti, A., Gratão, P. L., Gaziola, S. A., Lea, P. J., and Azevedo, R. A. (2006). Saccharopine dehydrogenase activity in the high-lysine opaque and floury maize mutants. Food Biotechnol. 20, 55-64. doi: 10.1080/ 08905430500524101

Posset, R., Opp, S., Struys, E. A., Völkl, A., Mohr, H., Hoffmann, G. F., et al. (2014). Understanding cerebral L-lysine metabolism: the role of L-pipecolate metabolism in Gcdh-deficient mice as a model for glutaric aciduria type I. J. Inher. Metab. Dis. 38, 265-272. doi: 10.1007/s10545-014-9762-z

Pratelli, R., and Pilot, G. (2014). Regulation of amino acid metabolic enzymes and transporters in plants. J. Exp. Bot. 65, 5535-5556. doi: 10.1093/jxb/eru320 
Ray, D. K., Gerber, J. S., MacDonald, G. K., and West, P. C. (2015). Climate variation explains a third of global crop yield variability. Nat. Commun. 6:5989. doi: $10.1038 /$ ncomms6989

Rodrigues, S. M., Andrade, M. O., Gomes, A. P. S., Da Matta, F. M., Baracat-Pereira, M. C., and Fontes, E. P. B. (2006). Arabidopsis and tobacco plants ectopically expressing the soybean antiquitin-like ALDH7 gene display enhanced tolerance to drought, salinity, and oxidative stress. J. Exp. Bot. 57, 1909-1918. doi: 10. 1093/jxb/erj132

Sacksteder, K. A., Biery, B. J., Morrell, J. C., Goodman, B. K., Geisbrecht, B. V., Cox, R. P., et al. (2000). Identification of the $\alpha$-aminoadipic semialdehyde synthase gene, which is defective in familial hyperlysinemia. Am. J. Genet. 66, 1736-1743. doi: $10.1086 / 302919$

Sauer, W. S., Opp, S., Hoffmann, G. F., Koeller, D. M., Okun, J. G., and Kolker, S. (2011). Therapeutic modulation of cerebral L-lysine metabolism in a mouse model for glutaric aciduria type I. Brain 134, 157-170. doi: 10.1093/brain/ awq269

Singh, S., Brocker, C., Koppaka, V., Chen, Y., Jackson, B. C., Matsumoto, A., et al. (2012). Aldehyde dehydrogenases in cellular responses to oxidative/electrophilic stress. Free Rad. Biol. Med. 56, 89-101. doi: 10.1016/j. freeradbiomed.2012.11.010

Slama, I., Abdelly, C., Bouchereau, A., Flowers, T., and Savoure, A. (2015). Diversity, distribution and roles of osmoprotective compounds accumulated in halophytes under abiotic stress. Ann. Bot. 115, 433-447. doi: 10.1093/aob/ mcu239

Sodek, L., and Wilson, C. M. (1970). Incorporation of leucine-C14 and lysine-C14 into protein in the developing endosperm of normal and opaque-2 corn. Arch. Biochem. Biophys. 140, 29-38. doi: 10.1016/0003-9861(70)90006-90008

Stepansky, A., Yao, Y., Tang, G., and Galili, G. (2005). Regulation of lysine catabolism in Arabidopsis through concertedly regulated synthesis of the two distinct gene products of the composite AtLKR/SDH locus. J. Exp. Bot. 56, 525-536. doi: 10.1093/jxb/eri031

Struys, E. A., and Jakobs, C. (2010). Metabolism of lysine in alpha-aminoadipic semialdehyde dehydrogenase-deficient fibroblasts: evidence for an alternative pathway of pipecolic acid formation. FEBS Lett. 584, 181-186. doi: 10.1016/j. febslet.2009.11.055

Struys, E. A., Jansen, E. E., and Salomons, G. S. (2014). Human pyrroline5-carboxylate reductase (PYCR1) acts on $\Delta 1$-piperideine-6-carboxylate generating L-pipecolic acid. J. Inherit. Metab. Dis. 37, 327-332. doi: 10.1007/s10545-013-9673-9674

Szabados, L., and Savoure, A. (2009). Proline: a multifunctional amino acid. Trends Plant Sci. 15, 89-97. doi: 10.1016/j.tplants.2009.11.009

Szekely, G., Abraham, E., Cseplo, A., Rigo, G., Zsigmond, L., Csiszar, J., et al. (2008). Duplicated P5CS genes of Arabidopsis play distinct roles in stress regulation and developmental control of proline biosynthesis. Plant J. 53, 11-28. doi: 10.1111/j.1365-313X.2007.03318.x

Szoke, A., Miao, G.-H., Hong, Z., and Verma, D. P. S. (1992). Subcellular location of $\Delta$ 1-Pyrroline-5-carboxylate reductase in root/nodule and leaf of soybean. Plant Physiol. 99, 1642-1649. doi: 10.1104/pp.99.4.1642

Van Wallendael, A., Soltani, A., Emery, N. C., Peixoto, M. M., Olsen, J., and Lowry, D. B. (2019). A Molecular view of plant local adaptation: incorporating stressresponse networks. Ann. Rev. Plant Biol. 70, 559-583. doi: 10.1146/annurevarplant-050718-100114
Verslues, P. E., and Sharma, S. (2010). Proline Metabolism and Its Implications for plant-environment interaction. Arabidopsis Book 3:e01401. doi: 10.1199/tab. 0140

Wang, C., Liu, R., Lim, G.-H., de Lorenzo, L., Yu, K., Zhang, K., et al. (2018). Pipecolic acid confers systemic immunity by regulating free radicals. Sci. $A d v$. 4:eaar4509. doi: 10.1126/sciadv.aar4509

Wang, Y., Schuck, S., Wu, J., Yang, P., Döring, A. C., Zeier, J., et al. (2018). A MPK3/6-WRKY33-ALD1-pipecolic acid regulatory loop contributes to systemic acquired resistance. Plant Cell 30, 2480-2494. doi: 10.1105/tpc.18. 00547

Wu, S., Tohge, T., Cuadros-Inostroza, A., Tong, H., Tenenboim, H., Kooke, R., et al. (2018). Mapping the Arabidopsis metabolic landscape by untargeted metabolomics at different environmental conditions. Mol. Plant 11, 118-134. doi: 10.1016/j.molp.2017.08.012

Yadav, A. K., Carroll, A. J., Estavillo, G. M., Rebetzke, G. J., and Pogson, B. J. (2019). Wheat drought tolerance in the field is predicted by amino acid responses to glasshouse-imposed drought. J. Exp. Bot. 70, 4931-4948. doi: 10.1093/jxb/ erz224

Yang, J. S., Hsu, J. W., Park, S. Y., Lee, S. Y., Li, J., Bai, M., et al. (2019). ALDH7A1 inhibits the intracellular transport pathways during hypoxia and starvation to promote cellular energy homeostasis. Nat. Commun. 10:4068. doi: 10.1038/ s41467-019-11932-11930

You, J., Zhang, Y., Liu, A., Li, D., Wang, X., Dossa, K., et al. (2019). Transcriptomic and metabolomic profiling of drought-tolerant and susceptible sesame genotypes in response to drought stress. BMC Plant Biol. 19:267. doi: 10.1186/s12870-019-1880-1

Yu, S., and Tian, L. (2018). Breeding major cereal grains through the lens of nutrition sensitivity. Mol. Plant 11, 23-30. doi: 10.1016/j.molp.2017. 08.006

Zhang, C. S., Lu, Q., and Verma, D. S. (1995). Removal of feedback inhibition of $\Delta$ 1-pyrroline-5-carboxylate synthetase, a bifunctional enzyme catalyzing the first two steps of proline biosynthesis in plants. J. Biol. Chem. 270, 20491-20496. doi: $10.1074 /$ jbc. 270.35 .20491

Zhou, H., Xia, D., and He, Y. (2020). Rice grain quality-traditional traits for high quality rice and health-plus substances. Mol. Breed. 40:1. doi: 10.1007/s11032019-1080- 1086

Zhou, J., Wang, X., Wang, M., Chang, Y., Zhang, F., Ban, S. Z., et al. (2018). The lysine catabolite saccharopine impairs development by disrupting mitochondrial homeostasis. J. Cell Biol. 218, 580-597. doi: 10.1083/jcb. 201807204

Conflict of Interest: The authors declare that the research was conducted in the absence of any commercial or financial relationships that could be construed as a potential conflict of interest.

Copyright (c) 2020 Arruda and Barreto. This is an open-access article distributed under the terms of the Creative Commons Attribution License (CC BY). The use, distribution or reproduction in other forums is permitted, provided the original author(s) and the copyright owner(s) are credited and that the original publication in this journal is cited, in accordance with accepted academic practice. No use, distribution or reproduction is permitted which does not comply with these terms. 